\title{
Microvesicles as a potential biomarker of neoplastic diseases and their role in development and progression of neoplasm
}

\author{
Magdalena Kajdos, Łukasz Janas, Dorota Kolasa-Zwierzchowska, Jacek R. Wilczyński, Tomasz Stetkiewicz
}

Gynecology and Gynecologic Oncology Department, Polish Mother's Memorial Hospital - Research Institute, Łódź, Poland

\begin{abstract}
Neoplastic diseases together with cardiovascular diseases are the most frequent causes of death in the Polish population. Cancers of reproductive organs with breast cancer are responsible for the highest morbidity and mortality in women suffering from neoplasm diseases. Asymptomatic dynamics of the development of a neoplasm and no deviations from the normal level of laboratory results contribute to the fact that malignant diseases are diagnosed too late. The aim of modern medicine is to diagnose cancer at the earliest stage, however, there is no sufficiently sensitive and specific biomarker which can be used for diagnostic, prognostic and therapeutic purposes.

Cellular interactions play the main role in the development, angiogenesis and invasiveness of a tumor. Recent research suggests the possibility of microvesicles (MVs) involvement in communication between cells. The MVs ability to fuse with various cells is used in cell-to-cell contact. Microvesicles cargo may include growth factors, their receptors, protease, adhesion molecules, signaling molecules and the sequence of DNA, mRNA, and micro-RNA. Larger quantities of MVs released from neoplastic cells affect both the local environment and systematic range causing metastases and progression. The research on molecular mechanisms of MVs' release and the presence of characteristic oncogenes in blood of patients with neoplasms is being carried out. Confirmation of MVs presence in patients' serum can potentially serve as useful information for therapeutic purposes and as the biomarker of a neoplastic disease.
\end{abstract}

Key words: microvesicles, neoplasm, biomarker, ovarian cancer.

\section{Introduction}

Ovarian activity to produce hormones decreases in the perimenopausal period and finally the release of hormones is inhibited. Lack of estradiol in postmenopausal women causes a high risk of multiple internal diseases such as arterial hypertension, coronary artery diseases, venous thromboembolism and arteriosclerosis. Moreover, 2/3 of postmenopausal women suffer from overweight and obesity. A lower level of physical activity, impropriate diet and lack of ovarian hormones cause lipid disorders and changes in the level of fractions of cholesterol. That fact and a high body mass index (BMI) increase a risk of arterial hypertension and arteriosclerosis. Stroke and heart failure are typical organ complications of hypertension and arteriosclerosis. They are a frequent cause of death in women after menopause. Cardiovascular diseases rank the first among the most common causes of death in women of the Polish population. Accidents and neoplastic diseases rank the second and the third, respectively.

Cancers of reproductive organs are frequently diagnosed in the peri- and postmenopausal period. Adeno- carcinoma of the uterus is the most common gynecological malignancy. Eighty percent of this type of cancer is diagnosed in women after menopause. Furthermore, hypertension, obesity and diabetes are risk factors for endometrial hyperplasia and cancer. Abnormal uterine bleeding (AUB) and abnormal sonographic features of the endometrium may suggest endometrial cancer. AUB and $\mathrm{ET}$ (endometrium thickness) $>4 \mathrm{~mm}$ indicate the need for endometrial biopsy and histological examination. When the endometrium is less than $4 \mathrm{~mm}$ in sonographic examination, a risk of endometrial cancer is lower. Invasive procedures allow to obtain endometrial tissue for histological examination. The result of examination makes it possible to choose appropriate treatment.

Similar proceedings are used to diagnose neoplasm of the vagina and vulva and to choose the proper treatment. Vulvae and vaginal cancer are frequently diagnosed in women after menopause. Clinical symptoms and vulvae and vaginal lesions require invasive methods of investigation such as a biopsy. Histopathological examination answers the question whether the lesion is benign or malignant. The result of histopathological 
examination is used to choose the appropriate treatment.

Cervical cancer is rarely diagnosed in postmenopausal women. A significant percentage of this cancer is diagnosed in younger women. In particular, it is a result of infection with high oncogenic types of human papillomavirus (HPV). Moreover, HPV infection may cause cancer of the vagina. This type of cancer is commonly diagnosed in postmenopausal women after treatment of cervical cancer.

Morbidity of ovarian cancer is higher in the peri- and postmenopausal period. Diagnosis of pre-neoplastic ovarian lesion and ovarian cancer is difficult because there are no specific diagnostic tools. Furthermore, the first symptoms appear in an advanced stage of diseases. An abnormal sonographic scan of the ovary and a higher level of CA 125 and HE4 may indicate a suspicion of malignant tumor. Surgery is the only procedure allowing to obtain ovarian tissue and ovarian tumor for histological examination.

The main purpose of research on early diagnosis of cancer is to find specific and sensitive biomarkers. Carcinogenesis is a complex process consisting of initiation, promotion and progression. Lesions previously occurring in a cell and then in the whole organism can last for years or months. The time beginning from changes in the DNA structure which initiate carcinogenesis and leads to a fully symptomatic disease depends on the type of a neoplasm and the type of a tissue it derives from. An important element of formation and progression of a neoplasm is ability to communicate, creating and transmitting information by neoplastic cells and ability of normal cells to respond to those signals [ 1 , $2,3-5]$. The exchange of messages between cells enables coordination of multicellular organism functioning. Cells can exchange the messages in three ways, in direct cell-to-cell contact (adhesion, juxtacrine interaction) and in an endocrine and a paracrine way [2, 4]. In an endocrine mechanism of communication, mediators are released into the blood and the bodily fluid as soluble or non-soluble molecules transported for example by proteins. After they have found the target cell they join the related receptor and activate a definite response. In a paracrine mechanism, the information is transmitted by changes in gradient concentration of soluble mediators in cells' microenvironment [1, 2]. The information is translated to activate the intracellular signaling network, which leads to changes in behavior of individual cells and their population $[2,6,7]$.

Recent research revealed a new model of cell-to-cell communication which depends on the release of microvesicles (MVs) via cells [1, 2, 4, 8-10]. Microvesicles cargo contains signaling packages to stimulate or inhibit target cells and may affect the properties of their surrounding microenvironment $[1,4,8]$. Moreover, MVs have ability to be transported long distances unless they are absorbed, undergo fusion or interact with target cells [2, 9-16]. There is some evidence indicating the involvement of MVs in physiological and pathological processes, such as immune response, carcinogenesis and angiogenesis [1, 3].

\section{Extracellular vesicles - exosomes, apoptotic bodies, microvesicles}

Microvesicles are one of several types of microparticles characterized by high levels of phosphatidylserine in membranes. They are released from different types of eukaryotic cells as a result of their activation by growth factor, cytokines or during apoptosis [17, 18]. Microvesicles, apoptotic bodies and exosomes are the most important and well-known particles, from which merely MVs and exosomes are used by the cells as carriers of information. The beginning of studies on these structures took place in the 60s of the last century, when Wolf described the microparticles in 1967 for the first time [19]. For years MVs had been considered as redundant microparticles - the form of waste products of life processes occurring in the cell. Nowadays their potential is recognized as a useful tool in diagnosis and treatment for many diseases for example, neoplasm [2, 10, 13-15, 20-22].

\section{Exosomes}

Exosomes were first described in 1981 [1, 23] as a cup-shaped structures $[1,24]$ ranging from 30 to $100 \mathrm{~nm}$ in size [1]. Other studies described them as oval-shaped structures and ranging from 40 to $100 \mathrm{~nm}$ in diameter [24]. Differences in exosome shapes can result from different methods of visualization and analysis. Their cup-shaped morphology can be a result of fixation procedures used for transmission electron microscopy (TEM), which is a standard method used to analyze the size of these microparticles [1, 25]. Exosomes observed by cryo-electron microscopy (cryo-EM) are visualized as oval-shaped vesicles [24, 26, 27].

Exosomes are the only vesicles composed of endosomal membranes [24]. This is because exosomes are released from multivesicular bodies (MVBs) which are endosomes transformed in intracellular processes. The fusion of MVBs membrane with plasma membrane leads to the release of their contents into the extracellular space and avoidance of MVBs degradation through lysosomal pathways [24]. Exosome membranes are characterized by a low level of phosphatidylserine exposure. However, these membranes contain other lipids, such as cholesterol, ceramide, sphingomyelin and lipids rafts [1, 24], glycolipid GM3 [24, 28, 29]. CD 63, CD81, CD 9, LAMP1, TSG101, Alix and HSC70 are distinguished as typical markers for exosomes [1, 12]. Recent studies on exosomes from cancer and immune cells reveal 
the ability of these structure to activate several cellular processes depending on their origin [1,30]. A multitude of activated pathways is a result of cellular interaction with exosomal molecules, including mRNA, microRNA (miRNA) and proteins (e.g., cytoskeleton proteins, heat shock proteins, adhesion molecules, proteins involved in signal transduction, transcription regulation and antigen presentation) [1]. Exosomes can be released from platelets, dendritic cells and cancer cells in laboratory conditions as well as in vivo, which confirms their presence in many biological fluids, including urine [31-33], plasma [34], peritoneal fluid [35], saliva [36], breast milk [37], bronchoalveolar lavage liquid [38], and amniotic fluid [39]. Accurate studies of their role in cancer progression, the development and improvement of collecting methods and isolation will enable the use of exosomes as potential biomarkers in the future.

\section{Apoptotic bodies}

Structures formed as a result of cell defragmentation in the process of programmed cell death (apoptosis) was first described in 1974 by Kerr [40] and named apoptotic bodies. Cellular organelles, fragments of DNA and histones surrounded by plasma membrane create apoptotic bodies with irregular shapes and ranging from 1 to $5 \mu \mathrm{m}$ in size [12]. Recent research has not revealed the role of those particles in intracellular communication as exosomes and MVs have. Some studies show that apoptotic bodies' cargo DNA and oncogenes play an important role in antigen presentation and immunosuppression [24, 41-44]. There is no appropriate and standard method of isolation that enables accurate analysis of apoptotic bodies and their role in eukaryotic organism functioning such as human body is.

\section{Microvesicles, ectosomes}

Many studies on MVs, which were described for the first time in 1967 by Wolf, have been carried out for the last two decades. Microvesicles are microparticles of different shapes and ranging from 100 to $1000 \mathrm{~nm}$ in size $[1,10,20]$. They are released from the outward surface of cells as a result of budding or blebbing of the plasma membrane $[1,10,20]$. Microvesicles are released from platelets, red blood cells, endothelial cells and carcinoma cells. This fact has been confirmed by many studies performed in many countries around the world [1, 22, 30, 45]. In tumor cells, MVs are released from the entire cell surface whereas in normal cells, there are selected areas of plasma membrane [1]. The membrane of tumor cells seems to be enriched by special lipids in the area of MVs release. Cholesterol is a lipid needed to form MVs and its lack causes inhibition of MVs production [46, 47]. Microvesicles are char- acterized by a high level of exposure to phosphatidylserine in their membranes, transported from the inner to outer surface of plasma membrane [1, 48]. Recent studies have not revealed typical surface markers for MVs. To signify those structures, flotillin-2, selectins, integrins, CD40, metalloproteinase can be used [1, 12, 49]. Proteins such as growth factors and their receptors, cytokines, chemokines [1, 4], enzymes, e.g. metalloproteinase, lipids, nucleic acid (mRNA, miRNA, ncRNA, DNA) $[1,21,50-54]$ can be transported inside MVs. The role of MVs in several processes in the human organism depends to a great extent on the type of cargo. Microvesicles take part in angiogenesis, carcinogenesis and immune response, because of their use as information carriers in intracellular communication. Moreover, they are said to transport information locally, which has been confirmed by their presence in urine or ascites, and systematically, that has been confirmed by their presence in circulating blood. The role of MVs in intracellular communication has been mostly investigated in carcinogenesis and inflammation. Interaction of intracellular communication may occur locally, regionally and systematically, which is often characterized by direct contact between MVs surface and the surface of a target cell $[1,55]$ or endocytosis [46]. The influence of MVs on target cells may lead to activation and discharge of paracellular bioactive cargos $[2,15,22,56]$. It can cause proteolytic remodeling and changes in properties of the microenvironment and changes in behavior of cells [2, 56]. Microvesicles circulating in blood stream and bodily fluids (saliva, urine, cerebrospinal fluid, peritoneal fluid) transfer onco-specified mediators called oncosomes [55]. The role of oncosomes is to transport oncoproteins [57], oncogenic transcript of mRNA [53], exchange oncogenic miRNA [2] and fragments of genomic DNA containing oncogenic sequences from cell to cell. Recent research has indicated the relationship between revealing and circulating in blood MVs and thrombosis, sepsis, coronary disease, multiple sclerosis, and some types of cancer [1, 58-62]. Researchers most accurately characterized MVs from platelet and endothelium and they noticed their increased level in several clinical disorders such as cardiovascular diseases (e.g., hypertension, atherosclerosis, congestive heart failure) [63-65], autoimmunological diseases (e.g., rheumatoid disease, vascular inflammation, diabetes type I) [66-69], hematological diseases and cerebral vessels diseases [70, 71]. Studies are still being carried out on the potential use of MVs as biomarkers which will be the gradient of the biological condition that changes according to disease progression or response to treatment. The idea of biomarkers is the ability to distinguish two similar conditions: pathological and physiological with the facility of collecting samples (e.g., saliva, blood or urine) for its identification. Routine non-invasive methods of indication should be precise, fast and potentially cheap [72]. 


\section{Microvesicles isolation}

The methods of isolation, characterization and enumeration of circulating MVs have not been validated completely. Several factors make particular steps of receiving and analysis of MVs difficult. Various studies have demonstrated that cancer-derived MVs have a lifespan of about 15-60 minutes in blood circulation $[55,73,74]$. It may result from rapid fusion and endocytosis by target cells [1]. Other research has disclosed their elimination via phagocytes due to a high level of phosphatidylserine in the outer surface leaflet [1]. The use of MVs released from tumor cells as biomarkers is restricted by a relatively small fraction of the total number of isolated MVs $[1,50]$. Those facts require defining pre-analytical procedures including anticoagulants, time from collecting samples to preliminary preparation (first centrifugation), temperature and time of storage and the methods of analysis.

For isolation MVs from blood sample, researchers have used methods based on their physical properties and biochemical features. The size and density are predominantly used as parameters for isolation. Precipitate with MVs is prepared as a result of centrifugations of platelet free plasma (PFP) in ultra-centrifuge at 20 000-60 $000 \times \mathrm{g}[2,55]$. Further isolation base on MVs size includes flotation in sucrose gradients and dielectrophoresis. Magnetophoretic sorting or immunoaffinity chromatography are used occasionally $[1,50]$.

The number of MVs in samples of the biological fluid is as important as their detection. Recent studies revealed the correlation between the quantity of MVs released via cells and the stage of a disease [1]. This fact may be used to make a rapid diagnosis, to assess prognosis and early detection of recurrence. That is why it is so important to form reliable and repeatable isolation methods. Flow cytometry is the most widely used method of MVs detection based on the measurement of MVs fluorescence previously marked with specific fluorescent antibodies. The imperfective aspect of this method is inability to notice vesicles (MVs and exosomes) smaller than $200 \mathrm{~nm}$ in diameter $[1,55]$, because in lower detection edges the appropriate signal is drowned out by instrumental noise. Potential difficulties may be reduced by calibration methods with special calibrations beads ranging from 0.2 to $2.0 \mu \mathrm{m}$ in diameter which are to decrease instrumental noise [55], however, it does not eliminate it totally. Moreover, there are no characteristic antibodies for microparticles. Annexin V was used to mark MVs, however, recent studies have revealed the presence of annexin $\mathrm{V}$-negative MVs $[1,30,75]$. It is necessary to use antibodies as markers for MVs on the basis of their cellular origin [1, 3, 76, 77]. The lack of a satisfactory method of analysis limits the use of ectosomes and exosomes in diagnostic and therapeutic processes. This fact and the potential of microparticles encourages to investigate and to create new methods of qualitative and quantitative evaluation.

\section{Role of microvesicles in carcinogenesis}

Microvesicles play an important role in the growth and progression of neoplastic disease [9, 20, 53, 57, 78]. Local tumor progression and invasion is possible due to protein modification of extracellular matrix. The degradation of extracellular matrix as a result of activation of the proteolytic cascade occurs due to lytic enzymes transported by cancer-derived MVs. The lytic enzymes include urokinase (UPA - plasminogen activator), metalloproteinases (MMPs) and cathepsins [79]. Released enzymes have the ability to damage the components of extracellular substance for example collagen IV and to reconstitute the basal membrane [80]. Moreover, it can lead to an increased tumor cell invasion and metastases [80]. Recent research has confirmed the presence of MVs including MMPs and UPA in the peritoneal fluid from patients with diagnosed ovarian cancer. A correlation between activation, gradient of increased enzymes and the stage of invasiveness has been observed [81, 82].

Microvesicles also play an important role in oncogenic transformation of cells and tumor acquisition of high invasive capabilities. Angelucci et al. confirmed that addition of MVs from PC3 cells (prostate cancer cells with high metastatic potential in humans) to LnCaP cells - low invasive prostate cancer cells, increased adhesion and invasive capabilities of LnCaP cells [80]. Carcinoma derived-MVs indicate abilities to transform normal fibroblasts and endothelial cells into cells characterized with higher ability of surviving and growth - typical tumor cell characteristics $[1,4,49]$. tTG (tissue - transglutaminase), enzyme transported via MVs, is considered to be responsible for those processes [49]. Another factor released as MVs cargo is an oncogenic form of the receptor for endothelial growth factor (EGFR). The oncogenic form of EGFR from glioma cancer cells contributes to carcinoma transformation of target normal glioma cells [57]. Moreover, MVs contain EGFR which has the ability to activate VEGF/VEGFR trail (vascular endothelial growth factor/vascular endothelial growth factor receptors trail) in endothelial cells that caused activation of angiogenesis [83]. The formation of new blood vessels is necessary for tumor progression, cell nutrition and metastases. The cells have the ability to transform proangiogenic stimuli with the use of various mechanisms, for example, stimulus factors may be released into tumor microenvironment or directly transmitted to endothelial cells $[1,4]$. Growth factors (VEGF, FGF) $[1,22,78]$ and proteolytic enzymes (e.g., MMPs, UPA) [22, 80, 84, 85] are the most common MVs proangiogenic cargo. Lytic enzymes promote new vessels formation due to the change of composition and the arrangement of extracellular matrix components. Studies have not excluded MVs influence on autocrine stimulation of endothelial cells to produce and release VEGF [83].

Those processes are possible due to carcinoma cell escaping capabilities from the host immune system 
and avoidance of apoptosis. Recent research has disclosed various mechanisms of interaction between cancer derived-MVs and the cells of the immune system. Microvesicles depending on origin (e.g., melanoma, colorectal carcinoma) indicate the ability to activate apoptosis in T-cell lymphocytes, inactivate of monocytes and inhibit cytokines release [1, 4, 86-88]. Tumor cells evade apoptosis by accumulation of factors responsible for programmed cell death in MVs and their release into extracellular matrix.

To sum up, cancer-derived MVs are unquestionably connected with the growth and progression of tumor cells. The mechanism of releasing each separate MVs generation is as important as their specific properties and composition associated with the type of cancer [2].

\section{Microvesicles as biomarkers of neoplastic disease}

Identification and documentation of MVs derived from spleen cells culture and lymphatic node cells from the patient suffering from Hodgkin disease in 1970 have started investigations and search for the use of those microparticles [89]. Microvesicles can be used as biomarkers for oncogenic diseases because tumor cells release high quantities of them in pathological processes including carcinogenesis and metastasis than in physiologic processes [1]. The research carried out on breast cancer cells revealed the deficiency of MVs released from normal population of breast cells MCF10A. Cells of invasive breast carcinoma such as MDA-MB 231 produced more MVs than cells with lower invasiveness (line - MCF7) [46, 90].

Transport inside MVs, exosomes and apoptotic bodies enables stabilization of the expression of microRNA [91] in the bloodstream and protects from enzyme degradation. RNases present in plasma have the capability to degrade $99 \%$ of RNA species within 15 minutes [92]. There are several families of miRNA. Those small fragments of single-stranded nucleic acid are composed of 19 to 25 nucleotides and respond to posttranscriptional gene expression. Their regulated function affects mainly cell proliferation and differentiation and cell cycle regulation [93]. Microvesicles can be potentially useful markers for an early oncological diagnosis because of the correlation between the evaluated level of a specific subset of miRNA and the type and stage of tumor, as well as stability of miRNA in the blood and other bodily fluids [1]. miRNA and mRNA molecules were identified in oncosomes deriving from the cells of pulmonary, stomach, colorectal, ovarian and glioma cancer [2, 46, 53, 94-99]. Microvesicles released from glioma cells contain mRNA promoting tumor growth and its invasiveness. They also enable immunoescape due to an inhibited immunoresponse [53]. mRNA activates cerebral endothelial cells to angiogenesis. The range of proangiogenic mRNA and
miRNA which have some influence on progression and metastasis, has been identified in CD105 positive cells of medullar kidney carcinoma [100].

An increased level of MVs transporting oncoproteins was confirmed in patients with a diagnosed prostate cancer, urinary bladder cancer and glioma. The culture of glandular prostate carcinoma cells indicates a higher production of MVs which enables progression. The elevated production can be associated with an increased oncogenic activity of protein kinase B (PKB/Akt), or result from stimulation with growth factors (EGF) [101]. An important element of this case is an activity of regulated protein DRF 3 (diaphanous related forming 3) because its increased level may be responsible for rate inhibition of MV formation and blebbing from cell membrane [101]. Moreover, the DRF 3 expression disappears during the progression of prostate cancer [101]. Researchers have isolated MVs from patients' urine with bladder carcinoma and have identified as their cargo eight proteins, whose level has been elevated in comparison to a sample from a healthy control group [1]. Change in protein composition of MVs isolated in urine has been observed in an early stage of a disease which could be used in earlier detection [102]. Recent studies disclose MVs, characterized by a higher concentration of CD133 and the transcript encoding the oncogenic form of EGFRvIII, in peripheral blood in a patient with glioblastoma [1, 51, 103]. Oncogenic receptors are important bioactive cargo transported by oncosomes and significantly modified microenvironment. Aggressive population of glioblastoma cells use EGFRvIII to influence non-aggressive cells. As a consequence, mitogen-activated protein kinase (MAPK) and Akt are released which leads to morphological transformation and an independent growth of a target cell [57]. Furthermore, the correlation between tumor removal, the decrease in the level of MVs and their total disappearance has been observed $[1,51,103]$.

Although the receptor for AXL carried inside MVs released in chronic lymphoblastic leukemia (CLL) can be used as a biomarker in the future. AXL-positive oncosomes condition the bone marrow remodeling in order to increase the disease progression [104]. The leukocyte proliferation has been inhibited in lymphoblastoma as the consequence of activity of immunesuppressing transmembrane protein - latent membrane protein LMP-I transported via MVs [105].

Another study has shown that MVs production via LOX melanoma cells is controlled by cascade involving ARF6 GTP-ase, phospholipase D, Erk and MLCK. This pathway causes phosphorylation of myosin light chain (MLC), which leads to MVs release, proteolysis and as a result, to an increase of cell invasiveness [106]. The presence of ARF6 has been also confirmed in MVs shed from breast cancer cells population [46]. Moreover, HER2/neu oncogenic receptor can be a component of those oncosomes [50,107, 108]. 
Tumor progression is possible because of a separate mechanism of MVs generation, their specific cargo and properties. Researchers have indicated the correlation between the quantity of derived-MVs and the stage of the neoplastic disease. The production of MVs which play some role in angiogenesis, cell migration and metastasis increases with its progression and invasiveness [15, 46]. In the neoplastic disease the MVs presence is as important as their concentration. An increased level of MVs has been observed in a patient with stage IV of gastric cancer in comparison with healthy controls. Moreover, it may be used in predicting metastasis formation [109]. The survival rate of patients with diffused breast cancer and pancreatic cancer has been estimated on the basis of MUC1 (epithelial mucin) expression and TF (tissue factor) activity in microvesicles identified in peripheral blood samples. The report revealed that the survival rate from 3 to 9 months was lower in patients with a higher level of TF activity and MUC1 expression when compared with a group with a low TF activity and no expression of MUC1 [110]. MVs analysis can be also useful in the detection of tumor recurrence $[50,111]$.

\section{Microvesicles as biomarkers of gynecological neoplastic diseases}

Cancer of reproductive organs together with breast cancer are the most frequent cause of death in women suffering from neoplastic diseases. 5500 women of the Polish population die from this group of malignant tumors every year. Adenocarcinoma of the uterus is the most frequent gynecological malignancy. The incidence of uterine cancer is relatively high (7\% in 2010 - Country Register of Neoplastic Diseases) and ranks the $4^{\text {th }}$ among the most common neoplastic diseases diagnosed in Polish women. Morbidity of uterine cervix neoplasm and ovarian cancer is lower. However, both types of malignancy frequently cause death. The incidence of neoplastic diseases increases with age. The peak of gynecological malignancy occurs in the peri- and postmenopausal period. It is important to remember that cancer can develop in younger women. Prognosis and results of treatment depend on the stage of the disease and early diagnosis. Some gynecological neoplasms can be diagnosed on gynecological examination (i.e. vulvae cancer). Basic diagnostic investigations, such as cytology, colposcopy, ultrasonography, $\mathrm{X}$-ray are frequently used to make the proper diagnosis. However, more advanced imaging methods, such as computer tomography, magnetic resonance and invasive examination (biopsy) have to be used. The use of cytology as a screening method of cervical cancer leads to a significant decrease in the incidence of this type of a neoplasm. Endometrial biopsy (pipelle, curettage) together with transvaginal ultrasound are used to diagnose uterine cancer. Endometrial biopsy is used to make histopathological diagnosis and allows to institute proper treatment quickly.

Specific diagnostic tools for identification of preneoplastic ovarian lesions and ovarian cancer at the lowest stage have not been found yet. Ovarian cancer is diagnosed late because the first symptoms appear in an advance stage of disease. As early diagnosis and proper treatment will prolong patients' life, it is important to find specific and sensitive biomarkers. The use of MVs as ovarian cancer biomarkers seems to be a good direction in making diagnosis.

Recent studies revealed MVs in the peritoneal fluid collected from the patient diagnosed with ovarian cancer. Microvesicles transport proteins such as enzymes and nucleic acid. Ovarian cancer cells release lytic enzymes which damage the components of extracellular substance and reconstitute the basal membrane. It leads to increased ability of tumor cells to invade and metastasize [1, 82]. Invasion of tumor cells through the extracellular matrix is essential for hematogenous, lymphatic, and peritoneal metastases [82]. Ascites produced in the case of ovarian malignancy contain high levels of proteolytic enzymes such as matrix metalloproteinase (MMP-2, MMP-9), and urokinase-type plasminogen activator (UPA) [82, 112, 113]. Moreover, these enzymes can be carried inside MVs $[1,82]$. Their activity is associated with the amount of MVs shed from tumor cells [1, 81]. Furthermore, an increased level of MVs in the peritoneal fluid correlates with tumor progression [1]. In addition, microvesicles may transport other proteinases a high level of which was confirmed in ovarian ascites. Some enzymes from the kallikreins family have invasive and stimulating properties that are used in tumor growth and metastases formation. Recent studies disclosed an overexpression of human kallikreins (hK) such as hK5, hK6, hK7, hK8, hK11, and hK14 [82, 114] in ovarian cancer. Moreover, hK5, hK6, hK10, and hK14 [82, 115-119] can be used as potential biomarkers of ovarian cancer.

Recent studies show the usefulness of MVs cargo miRNA in diagnosing ovarian cancer. The role of MVs is to protect miRNA from RNases [1, 92]. A significant number of miRNAs is deregulated and loses their suppressing function in cancer cells [1] that lead to tumor progression. miRNAs from the miR - 200 family are overexpressed in ovarian cancer [1]. Studies disclosed that a level of microparticles (MVs, exosomes) transported miRNA in the blood and peritoneal fluid is higher in women with malignant ovarian tumors in comparison to healthy patients or women with benign ovarian tumor [1, 94, 95]. Furthermore, the level of MVs and exosomes in blood correlated with the stage of diseases $[1,95]$. Moreover, it has been observed that an increase in the MVs level in serum overtakes the elevation of CA 125 [120].

Exosomes carried proteins play an important role in progression of ovarian cancer. TGF- $\beta$ and MAGE3/6 are 
cargo of exosomes isolated from plasma of patients suffering from malignant ovarian tumor [121]. In addition, there is an observed correlation between the amount of exosomal TGF- $\beta$ and MAGE3/ 6 and response to chemotherapy [121]. Failure of treatment and chemotherapy resistance are observed in patients with a higher level of exosomal proteins [121]. Subsequent findings demonstrated the application of MVs and exosomes as serum biomarkers of neoplastic diseases and their progression.

\section{Therapeutic use of microvesicles}

Although exosomes are well-known microparticles, further research on MVs is needed and may lead to a breakthrough in diagnostics and treatment of neoplastic diseases. Studies concerning the use of these two types of microparticles to create directed and personalized antineoplastic drugs are being carried out owing to their ability to transport drugs [17]. In the case of tumors indicating a higher level of oncogenic receptor HER2/ neu, for example ovarian cancer, breast cancer, stomach cancer and the presence of this protein in MVs collected from peripheral blood samples, the treatment blocking HER2 receptor can be instituted [50, 107]. Moreover, some studies have been undertaken to assess the possibility of placing mRNA and microRNA in MVs, which would enable the modification of the recipient's cell phenotype as a result of MVs fusion with its membrane.

\section{Conclusions}

The above results are promising and confirm the possibility to use MVs as biomarkers of a neoplastic disease. The analysis of MVs released by tumor cells into the bloodstream enables quick and repeated evaluation of carcinoma pathogenesis, biology, stage, progression, treatment response and recurrence. Noninvasiveness of the method allows to limit the number of surgical biopsies which may result in improper sample collection. Moreover, those microparticles may improve tumor diagnosis in which biopsy is contraindicated including ovary carcinoma, pancreas carcinoma and tumor of the nervous system. Collecting blood samples is not a burdening procedure for patients and its easiness additionally allows for a repeatable rate of MVs concentration and control of their level in the course of the disease. The analysis of received results enables to determine the dynamics of carcinogenesis and will have some influence on more effective and earlier detection of recurrences. The detection of MVs use as a routine examination requires to standardize and develop the methods of isolation and analysis of MVs from blood and bodily fluid samples. Further studies will not only enable early detection of a neoplastic disease, but will also contrib- ute to the improvement of treatment forms owing to new therapeutic methods.

\section{Disclosure}

Authors report no conflict of interest.

\section{References}

1. Giusti I, D’Ascenzo S, Dolo V. Microvesicles as Potential Ovarian Cancer Biomarkers. Biomed Res Int 2013; 2013: 703048.

2. Lee TH, D'Asti E, Magnus N, et al. Microvesicles as mediators of intercellular communication in cancer - the emerging science of cellular 'debris'. Semin Immunopathol 2011; 33: 455-467.

3. Enjeti AK, Lincz LF, Seldon M. Microparticles in health and disease. Semin Thromb Hemost 2008; 34: 683-691.

4. Rak J. Microparticles in cancer. Semin Thromb Hemost 2010; 36 : 888-906.

5. Heppner GH. Tumor cell societies. J Natl Cancer Inst 1989; 81: 648-649.

6. Citri A, Yarden Y. EGF-ERBB signalling: towards the systems level. Nat Rev Mol Cell Biol 2006; 7: 505-516.

7. Blume-Jensen P, Hunter T. Oncogenic kinase signalling. Nature 2001; 411: 355-365.

8. Schifferli JA. Microvesicles are messengers. Semin Immunopathol 2011; 33: 393-394.

9. Ratajczak J, Wysoczynski M, Hayek F, et al. Membrane-derived microvesicles: important and underappreciated mediators of cell-to-cell communication. Leukemia 2006; 20: 1487-1495.

10. Pilzer D, Gasser O, Moskovich O, et al. Emission of membrane vesicles: roles in complement resistance, immunity and cancer. Springer Semin Immunopathol 2005; 27: 375-387.

11. Théry C, Ostrowski $M$, Segura E. Membrane vesicles asconveyors of immune responses. Nat Rev Immunol 2009; 9: 581-593.

12. Mathivanan S, Ji H, Simpson RJ. Exosomes: extracellular organelles important in intercellular communication. J Proteomics 2010; 73 : 1907-1920.

13. Simons $M$, Raposo G. Exosomes-vesicular carriers for intercellular communication. Curr Opin Cell Biol 2009; 21: 575-581.

14. Camussi G, Deregibus MC, Bruno S, et al. Exosomes/microvesicles as a mechanism of cell-to-cell communication. Kidney Int 2010; 78: 838-848.

15. Muralidharan-Chari V, Clancy JW, Sedgwick A, Souza-Schorey C. Microvesicles: mediators of extracellular communication during cancer progression. J Cell Sci 2010; 123: 1603-1611.

16. Mause SF, Weber C. Microparticles: protagonists of a novel communication network for intercellular information exchange. Circ Res 2010; 107: 1047-1057.

17. Inal JM, Kosgodage $U$, Azam S, et al. Blood/plasma secretome and microvesicles. Biochim Biophys Acta 2013; 1834: 2317-2325.

18. Piccin A, Murphy WG, Smith OP. Circulating microparticles: pathophysiology and clinical implications. Blood Rev 2007; 21: 157-171.

19. Wolf $P$. The nature and significance of platelet products in human plasma. Br J Haematol 1967; 13: 269-288.

20. Al-Nedawi K, Meehan B, Rak J, Microvesicles: messengers and mediators of tumor progression. Cell Cycle 2009; 8: 2014-2018.

21. Simpson RJ, Lim JW, Moritz RL, Mathivanan S. Exosomes: proteomic insights and diagnostic potential. Export Rev Proteomics 2009; 6: 267-283.

22. Dolo V, D’Ascenzo S, Giusti I, et al. Shedding of membrane vesicles by tumor and endothelial cells. Ital J Anat Embryol 2005; 110: 127-133.

23. Trams EG, Lauter CJ, Salem N, Heine U. Exfoliation of membrane ectoenzymes in the form of micro-vesicles. Biochimica et Biophysica Acta 1981; 645: 63-70.

24. Meckes DG Jr, Raab-Traub N. Microvesicles and viral infection. J Virol 2011; 85: 12844-12854.

25. Théry C, Amigorena S, Raposo G, Clayton A. Isolation and characterization of exosomes from cell culture supernatants and biological fluids. Curr Protoc Cell Biol 2006; 3: 22. 
26. Conde-Vancells J, Rodriguez-Suarez E, Embade N, et al. Characterization and comprehensive proteome profiling of exosomes secreted by hepatocytes. J Proteome Res 2008; 7: 5157-5166.

27. Trajkovic K, Hsu C, Chiantia S, et al. Ceramide triggers budding of exosome vesicles into multivesicular endosomes. Science 2008; 319: 1244-1247.

28. Wubbolts R, Leckie RS, Veenhuizen PT, et al. Proteomic and biochemical analyses of human B cell-derived exosomes. Potential implications for their function and multivesicular body formation. J Biol Chem 2003; 278: 10963-10972.

29. de Gassart A, Geminard C, Fevrier B, et al. Lipid raft-associated protein sorting in exosomes. Blood 2003; 102: 4336-4344.

30. Gyorgy B, Modos K, Pallinger E, et al. Detection and isolation of cellderived microparticles are compromised by protein complexes due to shared biophysical parameters. Blood 2011; 117: e39-e48.

31. Conde-Vancells J, Rodriguez-Suarez E, Gonzalez E, et al. Candidate biomarkers in exosome-like vesicles purified from rat and mouse urine samples. Proteomics Clin Appl 2010; 4: 416-425.

32. Nilsson J, Skog J, Nordstrand A, et al. Prostate cancer-derived urine exosomes: a novel approach to biomarkers for prostate cancer. $\mathrm{Br} J$ Cancer 2009; 100: 1603-1607.

33. Pisitkun T, Shen RF, Knepper MA. Identification and proteomic profiling of exosomes in human urine. Proc Natl Acad Sci U S A 2004; 101: 13368-13373.

34. Orozco AF, Jorgez CJ, Ramos-Perez WD, et al. Placental release of distinct DNA-associated micro-particles into maternal circulation: reflective of gestation time and preeclampsia. Placenta 2009; 30: 891-897.

35. Andre F, Schartz NE, Movassagh M, et al. Malignant effusions and immunogenic tumour-derived exosomes. Lancet 2002; 360: 295-305.

36. Michael A, Bajracharya SD, Yuen PS, et al. Exosomes from human saliva as a source of microRNA biomarkers. Oral Dis 2010; 16: 34-38.

37. Admyre C, Johansson SM, Qazi KR, et al. Exosomes with immune modulatory features are present in human breast milk. J Immunol 2007; 179: 1969-1978.

38. Prado N, Marazuela EG, Segura E, et al. Exosomes from bronchoalveolar fluid of tolerized mice prevent allergic reaction. J Immunol 2008; 181: 1519-1525.

39. Keller S, Rupp C, Stoeck A, et al. CD24 is a marker of exosomes secreted into urine and amniotic fluid. Kidney Int 2007; 72: 1095-1102.

40. Kerr JF, Wyllie AH, Currie AR. Apoptosis: a basic biological phenomenon with wide-ranging implications in tissue kinetics. Br J Cancer 1972; 26: 239-257.

41. Bellone M, lezzi G, Rovere P, et al. Processing of engulfed apoptotic bodies yields T cell epitopes. J Immunol 1997; 159: 5391-5399.

42. Bergsmedh A, Szeles A, Henriksson M, et al. Horizontal transfer of oncogenes by uptake of apoptotic bodies. Proc Natl Acad Sci U S A 2001; 98: 6407-6411

43. Cocca BA, Cline AM, Radic MZ. Blebs and apoptotic bodies are B cell autoantigens. J Immunol 2002; 169: 159-166.

44. Holmgren L, Szeles A, Rajnavölgyi E, et al. Horizontal transfer of DNA by the uptake of apoptotic bodies. Blood 1999; 93: 3956-3963.

45. Taraboletti G, D'Ascenzo S, Borsotti P, et al. Shedding of the matrix metalloproteinases MMP-2, MMP-9, and MT1-MMP as membrane vesicle-associated components by endothelial cells. Am J Pathol 2002; 160: 673-680.

46. D'Souza-Schorey C, Clancy JW. Tumor-derived microvesicles: shedding light on novel microenvironment modulators and prospective cancer biomarkers. Genes Dev 2012; 26: 1287-1299.

47. Del Conde I, Shrimpton CN, Thiagarajan P, Lopez JA. Tissue-factorbearing microvesicles arise from lipid rafts and fuse with activated. Blood 2005; 106: 1604-1611.

48. Hugel B, Martínez MC, Kunzelmann C, Freyssinet JM. Membrane microparticles: two sides of the coin. Physiology 2005; 20: 22-27.

49. Antonyak MA, Li B, Boroughs LK, et al. Cancer cell derived microvesicles induce transformation by transferring tissue transglutaminase and bronectin to recipient cells. Proc Natl Acad Sci U S A 2011; 108: $4852-4857$.

50. Hugel B, Martínez MC, Kunzelmann C, Freyssinet JM. Membrane microparticles: two sides of the coin. Physiology 2005; 20: 22-27.
51. D'Souza-Schorey C, Clancy JW. Tumor-derived microvesicles: shedding light on novel microenvironment modulators and prospective cance biomarkers. Genes Dev 2012; 26: 1287-1299.

52. Valadi $\mathrm{H}$, Ekstrom K, Bossios A, et al. Exosome-mediated transfer of mRNAs and microRNAs is a novel mechanism of genetic exchange between cells. Nat Cell Biol 2007; 9: 654-659.

53. Skog J, Wurdinger T, van Rijn S, et al. Glioblastoma microvesicles transport RNA and proteins that promote tumour growth and provide diag nostic biomarkers. Nat Cell Biol 2008; 10: 1470-1476.

54. Pisetsky DS, Gauley J, Ullal AJ. Microparticles as asource of extracellular DNA. Immunol Res 2011; 49: 227-234.

55. Jayachandran $M$, Litwiller RD, Owen WG et al Characterization of blood borne microparticles as markers of premature coronary calcification in newly menopausal women. Am J Physiol Heart Circ Physio 2008; 295: H931-H938.

56. Hendrix A, WestbroekW, Bracke $M$, et al. An ex(o)citing machinery fo invasive tumor growth. Cancer Res 2010; 70: 9533-9537.

57. Al-Nedawi K, Meehan B, Micallef J, et al. Intercellular transfer of the oncogenic receptor EGFRvIII by microvesicles derived from tumor cells. Nat Cell Biol 2008; 10: 619-624.

58. Toth B, Liebhardt S, Steinig K, et al. Platelet-derived microparticles and coagulation activation in breast cancer patients. Thromb Haemost 2008; 100: 663-669.

59. Zwicker Jl, Liebman HA, Neuberg D, et al. Tumor-derived tissue factorbearing microparticles are associated with venous thromboembolic events in malignancy. Clin Cancer Res 2009; 15: 6830-6840.

60. VanWijk MJ, VanBavel E, Sturk A, Nieuwland R. Microparticles in cardiovascular diseases. Cardiovasc Res 2003; 59: 277-287.

61. Berckmans RJ, Nieuwland R, Böing AN, et al. Cell-derived microparticles circulate in healthy humans and support low grade thrombin generation. Thromb Haemost 2001; 85: 639-646

62. Joop K, Berckmans RJ, Nieuwland R, et al. Microparticles from patients with multiple organ dysfunction syndrome and sepsis support coagulation through multiple mechanisms. Thromb Haemost 2001; 85 810-820

63. Nozaki T, Sugiyama S, Sugamura K, et al. Prognostic value of endothelial microparticles in patients with heart failure. Eur J Heart Fail 2010 12: $1223-1228$.

64. Chironi G, Simon A, Hugel B, et al. Circulating leukocyte-derived microparticles predict subclinical atherosclerosis burden in asymptomatic subjects. Arterioscler Thromb Vasc Biol 2006; 26: 2775-2780.

65. Preston RA, Jy W, Jimenez JJ, et al. Effects of severe hypertension on en dothelial and platelet microparticles. Hypertension 2003; 41: 211-217.

66. Minagar A, Jy W, Jimenez JJ, et al. Elevated plasma endothelial microparticles in multiple sclerosis. Neurology 2001; 56: 1319-1324.

67. Sabatier F, Darmon P, Hugel B, et al. Type 1 and type 2 diabetic patients display different patterns of cellular microparticles. Diabetes 2002; 51: 2840-2845.

68. Brogan PA, Dillon MJ. Endothelial microparticles and the diagnosis of the vasculitides. Int Med 2004; 43: 1115-1119.

69. Knijff-Dutmer EA, Koerts J, Nieuwland R, et al. Elevated levels of platelet microparticles are associated with disease activity in rheumatoid arthritis. Arthritis Rheum 2002; 46: 1498-1503.

70. Simak J, Holada K, Risitano AM, et al. Elevated circulating endothelia membrane microparticles in paroxysmal nocturnal haemoglobinuria. Br J Haematol 2004; 125: 804-813.

71. Jung KH, Chu K, Lee ST, et al. Circulating endothelial microparticles as a marker of cerebrovascular disease. Ann Neurol 2009; 66: 191-199.

72. Joos TO, Bachmann J. The promise of biomarkers: research and applications. Drug Discovery Today 2005; 10: 615-616.

73. Gross PL, Furie BC, Merrill-Skoloff G, et al. Leukocyte-versus microparticle-mediated tissue factor transfer during arteriolar thrombus development. J Leukoc Biol 2005; 78: 1318-1326.

74. Thomas GM, Panicot-Dubois L, Lacroix R, et al. Cancer cell-derived microparticles bearing P-selectin glycoprotein ligand 1 accelerate thrombus formation in vivo. J Exp Med 2009; 206: 1913-1927.

75. Connor DE, Exner T, Ma DD, Joseph JE. The majority of circulating platelet-derived microparticles fail to bind annexin V, lack phospholipiddependent procoagulant activity and demonstrate greater expression of glycoprotein Ib. Thromb Haemost 2010; 103: 1044-1052. 
76. Flaumenhaft R, Dilks JR, Richardson J, et al. Megakaryocyte-derived microparticles: direct visualization and distinction from platelet-derived microparticles. Blood 2009; 113: 1112-1121.

77. Cerri C, Chimenti D, Conti I, et al. Monocyte/macrophage-derived microparticles upregulate inflammatory mediator synthesis by human airway epithelial cells. J Immunol 2006; 177: 1975-1980.

78. Taraboletti G, D'Ascenzo S, Giusti I, et al. Bioavailability of VEGF in tumor-shed vesicles depends on vesicle burst induced by acidic $\mathrm{pH}$. Neoplasia 2006; 8: 96-103.

79. Inal JM, Ansa-Addo EA, Stratton D, et al. Microvesicles in health and disease. Arch Immunol Ther Exp (Warsz) 2012; 60: 107-121.

80. Angelucci A, D'Ascenzo S, Festuccia C, et al. Vesicleassociated urokinase plasminogen activator promotes invasion in prostate cancer cell lines. Clin Exp Metastasis 2000; 18: 163-170.

81. Ginestra A, La Placa MD, Saladino F, et al. The amount and proteolytic content of vesicles shed by human cancer cell lines correlates with their in vitro invasiveness. Anticancer Res 1998; 18: 3433-3437.

82. Graves LE, Ariztia EV, Navari JR, et al. Proinvasive properties of ovarian cancer ascites-derived membrane vesicles. Cancer Res 2004; 64 7045-7049.

83. Al-Nedawi K, Meehan B, Kerbel RS, et al. Endothelial expression of au tocrine VEGF upon the uptake of tumor-derived microvesicles containing oncogenic EGFR. Proc Natl Acad Sci U S A 2009; 106: 3794-3799.

84. Dolo V, Ginestra A, Ghersi G, et al. Human breast carcinoma cells cultured in the presence of serum shed membrane vesicles rich in gelatinolytic activities. J Submicrosc Cytol Pathol 1994; 26: 173-180.

85. Giusti I, D’Ascenzo S, Millimaggi D, et al. Cathepsin B mediates the $\mathrm{pH}$-dependent proinvasive activity of tumor-shed microvesicles. Neoplasia 2008; 10: 481-488.

86. Huber V, Fais S, lero M, et al. Human colorectal cancer cells induce T-cell death through release of proapoptotic microvesicles: role in immune escape. Gastroenterology 2005; 128: 1796-1804.

87. Jeong WK, Wieckowski E, Taylor DD, et al. Fas ligand-positive membranous vesicles isolated from sera of patients with oral cancer induce apoptosis of activated T lymphocytes. Clin Cancer Res 2005; 11: 1010-1020.

88. Valenti R, Huber V, lero M, et al. Tumor-released microvesicles as vehicles of immunosuppression. Cancer Res 2007; 67: 2912-2915.

89. Friend C, Marovitz W, Henie G, et al. Observations on cell lines derived from a patient with Hodgkin's disease. Cancer Res 1978; 38 2581-2591.

90. D'Souza-Schorey C, Chavrier P. ARF proteins: Roles in membrane traf fic and beyond. Nat Rev Mol Cell Biol 2006; 7: 347-358.

91. Kobayashi E, Ueda Y, Matsuzaki S, et al. Biomarkers for screening, di agnosis and monitoring of ovarian cancer. Cancer Epidemiol Biomarkers Prev 2012; 21: 1902-1912.

92. Mikaelian I, Scicchitano M, Mendes O, et al. Frontiers in preclinica safety biomarkers: microRNAs and messenger RNAs. Toxicol Patho 2013; 41: 18-31.

93. He L, Hannon GJ. microRNAs: small RNAs with a big role in gene regulation. Nat Rev Genetics 2004; 5: 522-531.

94. Taylor DD, Gercel-Taylor C. microRNA signatures of tumor-derived exosomes as diagnostic biomarkers of ovarian cancer. Gynecol Oncol 2008; 110: 13-21

95. Resnick KE, Alder H, Hagan JP, et al. The detection of differentially expressed microRNAs from the serum of ovarian cancer patients using a novel real-time PCR platform. Gynecol Oncol 2009; 112: 55-59.

96. Baran J, Baj-Krzyworzeka M, Weglarczyk K, et al. Circulating tumourderived microvesicles in plasma of gastric cancer patients. Cancer Immunol Immunother 2009; 59: 841-850.

97. Hong BS, Cho JH, Kim H, et al. Colorectal cancer cellderived microvesicles are enriched in cell cycle-related mRNAs that promote proliferation of endothelial cells. BMC Genomics 2009; 10: 556.

98. Rabinowits G, Gercel-Taylor C, Day JM, et al. Exosomal microRNA: a diagnostic marker for lung cancer. Clin Lung Cancer 2009; 10: 42-46.

99. Ohshima K, Inoue K, Fujiwara A, et al. Let-7 microRNA family is selectively secreted into the extracellular environment via exosomes in a metastatic gastric cancer cell line. PLoS ONE 2010; 5: e13247.

100. Grange C, Tapparo M, Collino F, et al. Microvesicles released from human renal cancer stem cells stimulate angiogenesis and formation of lung premetastatic niche. Cancer Res 2011; 71: 5346-5356.
101. Di Vizio D, Kim J, Hager MH, et al. Oncosome formation in prostate cancer: association with a region of frequent chromosomal deletion in metastatic disease. Cancer Res 2009; 69: 5601-5609.

102. Smalley DM, Sheman NE, Nelson K, Theodorescu D. Isolation and identification of potential urinary microparticle biomarkers of bladder cancer. J Proteome Res 2008; 7: 2088-2096.

103. Huttner HB, Janich P, Köhrmann M, et al. The stem cell marker prominin-1/CD133 on membrane particles in human cerebrospinal fluid offers novel approaches for studying central nervous system disease. Stem Cells 2008; 26: 698-705.

104. Ghosh AK, Secreto CR, Knox TR, et al. Circulating microvesicles in B-cell chronic lymphocytic leukemia can stimulate marrow stromal cells: implications for disease progression. Blood 2010; 115: 17551764 .

105. Flanagan J, Middeldorp J, Sculley T. Localization of the Epstein-Barr virus protein LMP 1 to exosomes. J Gen Virol 2003; 84: 1871-1879.

106. Muralidharan-Chari V, Clancy J, Plou C, et al. ARF6-regulated shedding of tumor cell-derived plasma membrane microvesicles. Curr Biol 2009; 19: 1875-1885.

107. Arteaga CL, Sliwkowski MX, Osborne CK, et al. Treatment of HER2positive breast cancer: current status and future perspectives. Nat Rev Clin Onocl 2011; 9: 16-32.

108. Koga K, Matsumoto K, Akiyoshi T, et al. Purification, characterization and biological significance of tumor-derived exosomes. Anticancer Res 2005; 25: 3703-3707

109. Kim HK, Song KS, Park YS, et al. Elevated levels of circulating platelet microparticles, VEGF, IL-6 and RANTES in patients with gastric cancer: possible role of a metastasis predictor. Eur J Cancer 2003; 39: 184191.

110. Tesselaar ME, Romijn FP, van der Linden IK, et al. Microparticle associated tissue factor activity: a link between cancer and thrombosis? J Thromb Haemost 2007; 5: 520-527.

111. Colombo E, Borgiani B, Verderio C, Furlan R. Microvesicles: novel biomarkers for neurological disorders. Front Physiol 2012; 3: 63.

112. Young TN, Rodriguez GC, Rinehart AR, et al. Characterization of gelatinases linked to extracellular matrix invasion in ovarian adenocarcinoma: purification of matrixmetalloproteinase 2. Gynecol Oncol 1996; 62: 89-99.

113. Dolo V, D’Ascenzo S, Violini S, et al. Matrix-degrading proteinases are shed in membrane vesicles by ovarian cancer cells in vivo and in vitro. Clin Exp Metastasis 1999; 17: 131-140.

114. Yousef GM, Polymeris ME, Yacoub GM, et al. Parallel overexpression of sevenkallikrein genes in ovarian cancer. Cancer Res 2003; 63: 22232227

115. Yousef GM, Polymeris ME, Grass L, et al. Human kallikrein 5: a potential novel serum biomarker for breast and ovarian cancer. Cancer Res 2003; 63: 3958-3965.

116. Diamandis EP, Scorilas A, Fracchioli S, et al. Human kallirein 6 (hK6): a new potential serum biomarker for diagnosis and prognosis of ovarian cancer. J Clin Oncol 2003; 21: 1035-1043.

117. Magklara A, Mellati AA, Wasney GA, et al. Characterization of the enzymatic activity of human kallikrein 6: autoactivation, substrate specificity, and regulation by inhibitors. Biochem Biophys Res Commun 2003; 307: 948-955

118. Luo LY, Katsaros D, Scorilas A, et al. The serum concentration of human kallikrein 10 represents a novel biomarker for ovarian cancer diagnosis and prognosis. Cancer Res 2003; 63: 807-811.

119. Borgoño CA, Grass L, Soosaipillai A, et al. Human kallikrein 14: a new potential biomarker for ovarian and breast cancer. Cancer Res 2003; 63: 9032-9041.

120. Ginestra A, Miceli D, Dolo V, et al. Membrane vesicles in ovarian cancer fluids: a new potential marker. Anticancer Res 1999; 19: 3439-3445.

121. Szajnik M, Derbis M, Lach M, et al. Exosomes in Plasma of Patients with Ovarian Carcinoma: Potential Biomarkers of Tumor Progression and Response to Therapy. Gynecol Obstet (Sunnyvale) 2013; Suppl 4: 3. 